\title{
Remote control of brain angiotensin II levels by angiotensin receptor blockers
}

\author{
Masaki Mogi and Masatsugu Horiuchi \\ Hypertension Research (2010) 33, 116-117; doi:10.1038/hr.2009.209; published online 11 December 2009
}

$\mathrm{T}$ he contribution of the renin-angiotensin-aldosterone system (RAS) to central nervous system (CNS) disorders has been studied extensively. Because all components of the RAS exist in the CNS, angiotensin (Ang) II is produced and functions as a local RAS. Therefore, the central action of Ang II is considered to be induced by generated brain Ang II. Central Ang II controls not only water and sodium intake but also the pathophysiology of CNS diseases, such as stroke and neurodegenerative diseases. Blockade of RAS by Ang II type-1 ( $\left.\mathrm{AT}_{1}\right)$ receptor blockers (ARBs) or angiotensin-converting enzyme (ACE) inhibitors has been widely used as an antihypertensive treatment and is expected to protect target organs, including the brain. Because oral administration of ARBs prevents the central effect of Ang II through hypothalamic-pituitary-adrenal stimulation and modulation of the sympathoadrenal response, the benefits of 'oral' administration of ARBs are considered to be partly due to its penetration through the bloodbrain barrier (BBB). However, a direct brainprotective effect of $A R B s$ across the $B B B$ is not totally clear because there are no remarkable differences in brain-protective effects among ARBs in spite of the different abilities of ARBs to cross the BBB. Moreover, the relationship between Ang II levels in the plasma and in the brain tissue is also enigmatic. Mikami et al. ${ }^{1}$ reported 24 years ago that intravenous Ang II infusion significantly augmented Ang II immunoreactivity in plasma but had no effect in cerebrospinal fluid, indicating that regulation of local RAS

Dr M Mogi is at the Department of Molecular Cardiovascular Biology and Pharmacology, Ehime University Graduate School of Medicine, Shitsukawa, Tohon, Ehime 791-0295, Japan.

E-mail: mmogi@m.ehime-u.ac.jp is independent of the circulating Ang II level by the BBB. Moreover, the change in Ang II levels in the brain after systemic administration of ARBs has not been thoroughly investigated.

The study by Pelisch et al. ${ }^{2}$ demonstrates that systemic treatment with ARB candesartan reduces brain Ang II levels with a local brain RAS activity. The authors measured brain and plasma Ang II levels by the radioimmunoassay method originally generated by their group. ${ }^{3}$ Similar to the previous study, Ang II-infused mice demonstrated an increase in plasma Ang II, but not in brain Ang II. In contrast, systemic candesartan treatment remarkably reduced brain Ang II levels without any change in plasma Ang II. Previously, their research group also demonstrated that systemic treatment with another $\mathrm{ARB}$, olmesartan, reduced the reactive upregulation of brain Ang II in Sprague-Dawley rats after middle cerebral artery occlusion. ${ }^{4}$ However, the mechanism leading to reduced brain Ang II after ARB treatment has not been investigated. In this issue, Pelisch et al. ${ }^{2}$ indicate that systemic treatment with $\mathrm{ARB}$ remarkably attenuates the mRNA expression of both angiotensinogen and ACE in the brain. BBB permeability with or without Ang II infusion was also investigated using horseradish peroxidase-conjugated tetramethylbenzine. The authors considered the fact that candesartan does not penetrate into the brain. These findings reveal the important result that systemic treatment with $\mathrm{ARB}$ remotely regulates brain Ang II. However, several questions are raised and should be resolved in the future. (1) Does candesartan penetrate into the brain at all? Pelisch et al. ${ }^{2}$ used an Ang II-infused model to assess the penetration of this ARB; however, its penetration is unknown in other models, such as mice with diabetes mellitus, a highsalt diet or obesity. Moreover, candesartan could modulate the brain RAS even at a level undetectable by this method. Therefore, it remains unclear whether candesartan needs to cross the BBB to exert its action. (2) Was this effect specific to candesartan, or was it a class effect for ARBs in general? Considering the results of their previous report using olmesartan, ${ }^{4}$ ARBs may reduce brain Ang II levels as a class effect. However, comparisons of drug-specific effects among different ARBs should be investigated. (3) What is the detailed mechanism of the reduction of brain RAS by candesartan? Is it actually remotely controlled? Otherwise, $\mathrm{AT}_{1}$ receptor blockade in the cerebrovasculature by candesartan may induce a decrease in expression of angiotensinogen and ACE in brain tissue. Furthermore, a regional analysis of Ang II distribution is also expected. The answers to these questions would clarify the beneficial role of ARBs in CNS disorders.

Finally, this study provides us with a new mechanistic insight into antihypertensive treatment by RAS blockade. Brain Ang II regulates blood pressure and circulating Ang II through the sympathetic nerve system. This finding indicates that systemic treatment with ARBs caused reduced brain RAS activity. In the future, new approaches to study the relationship between brain RAS and regulation of blood pressure by RAS blockade will be expected.

\section{CONFLICT OF INTEREST}

The authors declare no conflict of interest.

\section{ACKNOWLEDGEMENTS}

This study was supported by grants from the Ministry of Education, Science, Sports and Culture 
of Japan to MH and the Novartis Foundation for Gerontological Research to MM.

1 Mikami $H$, Suzuki $H$, Smeby RR, Ferrario CM. Cerebrospinal fluid angiotensin II immunoreactivity is not derived from the plasma. Hypertension 1985; 7: 65-71.

2 Pelisch N, Hosomi N, Ueno M, Masugata H, Murao K, Hitomi $H$, Nakano D, Kobori $H$, Nishiyama A, Kohno M. Systemic candesartan reduces brain angiotensin II via downregulation of brain renin-angiotensin system. Hypertens Res 2009 (e-pub ahead of print 27 November 2009; doi:10.1038/hr.2009.200).
3 Nishiyama A, Seth DM, Navar LG. Renal interstitial fluid concentrations of angiotensins I and II in anesthetized rats. Hypertension 2002; 39: 129-134.

4 Hosomi N, Nishiyama A, Ban CR, Naya T, Takahashi T, Kohno M, Koziol JA. Angiotensin type 1 receptor blockage improves ischemic injury following transient focal cerebral ischemia. Neuroscience 2005; 134: 225-231. 